\title{
A CORRELAÇÃO ENTRE O NÍVEL DE CIRCUNFERÊNCIA DE CINTURA E O GRAU DE ATIVIDADE FÍSICA
}

\section{The correlation between waist circumference level and physical activity degree}

\author{
Marcus Vinicius Accetta Vianna, Ignácio A. Seixas-da-Silva, André Luiz Marques Gomes
}

\section{Resumo}

A prevalência de obesidade tem aumentado, a taxas alarmantes, em todo o mundo, passando a ser considerada uma epidemia global. A atividade física é o componente mais variável do gasto energético diário. $\mathrm{O}$ objetivo do presente estudo, então, foi correlacionar o grau de atividade física com o nível de circunferência de cintura, verificando se os indivíduos mais ativos fisicamente possuem menores valores de circunferência de cintura. A amostra foi composta por 40 indivíduos (24 homens e 16 mulheres) avaliados no Barra Shopping (RJ). O grau de atividade física foi classificado através do questionário de atividade física habitual. O perfil do estilo de vida foi avaliado, considerando cinco fatores individuais relacionados ao bem-estar: nutrição, atividade física, comportamento preventivo, relacionamento social e controle do estresse. Sobrepeso e obesidade foram analisados através do índice de massa corporal. Foi observada uma alta prevalência de sobrepeso entre os homens (58,33\%). A prevalência de circunferência de cintura nível I e nível II, entre as mulheres, foi de $37,5 \%$ e entre os homens, de $33,32 \%$. Os componentes nutrição e atividade física tiveram um perfil negativo, tanto em homens, quanto em mulheres. Foi encontrada uma correlação negativa entre o nível de circunferência de cintura e o grau de atividade física, tanto em homens $(r=-0,55 ; p<0,01)$, quanto em mulheres $(r=-0,55 ; p<0,05)$, sugerindo que a atividade física pode influenciar os valores de circunferência de cintura.

Palavras-chave: Atividade Física, Circunferência de Cintura, Índice de Massa Corporal, Sobrepeso, Obesidade.

\begin{abstract}
Obesity prevalence has increased at alarming rates in all parts of the world, being considered as a global epidemic. The physical activity is the most variable component in the daily energy cost. Due to this, the aim of the study was to correlate physical activity degree with waist circumference level, verifying if more physically active people have less waist circumference. The sample was composed of 40 individuals (24 men and 16 women) evaluated in Barra Shopping (RJ). The physical activity degree was evaluated through the questionnaire of habitual physical activities. The lifestyle profile was analyzed considering five individual factors related to well-being (nutrition, physical activity, preventive behavior, social relationships and stress control). Overweight and obesity were evaluated through the body mass index (BMI). It was observed a high prevalence of overweight among the men (58.33\%). The prevalence of waist circumference level I and level II was of $37.5 \%$ among the women, and $33.32 \%$ among the men. The nutrition and physical activity components had a negative profile in both men and women. It was found a negative correlation between waist circumference level and physical activity degree in both men $(r=-0.55 ; p<0.01)$ and women $(r=-0.55 ; p<0.05)$, suggesting that physical activity can influence waist circumference scores.
\end{abstract}

Key words: Physical Activity, Waist Circumference, Body Mass Index, Overweight, Obesity.

Laboratório de Fisiologia do Exercício (LAFIEX ) - Universidade Estácio de Sá - Campos dos Goytacazes e Niterói - RJ - Brasil.

Revista de Educação Física 2008 Set; 142:42-49. Rio de Janeiro (RJ) - Brasil.

Recebido em 20.02.2008. Aceito em 17.06.2008. 


\section{INTRODUÇÃO}

Evidências sugerem que as prevalências de sobrepeso e obesidade têm aumentado a taxas alarmantes, incluindo países desenvolvidos e em desenvolvimento, sendo considerada uma epidemia global ${ }^{(1,2)}$.

A obesidade pode ser definida como o excesso de tecido adiposo, a partir do qual os riscos para a saúde começam a aumentar, consistindo em um percentual de gordura corporal anormalmente elevado, que pode ser generalizado ou localizado ${ }^{(3)}$.

De forma simplista, pode-se dizer que a obesidade resulta de um balanço energético positivo, ou seja, de um desequilíbrio entre a energia ingerida e a energia gasta, podendo ocorrer em função de um aumento da ingestão energética, de uma redução do gasto energético total ou pela combinação dos dois fatores ${ }^{(3-5)}$.

Martins e Rodrigues dos Santos (6) afirmam que indivíduos apresentando maiores valores de ingestão energética e menores índices de atividade física de lazer evidenciavam maiores índices de massa gorda.

Com a modernidade e com o avanço tecnológico, as pessoas, em geral, vêm se tornando mais sedentárias. Esta contemporânea redução no dispêndio energético, além de hábitos alimentares inadequados, têm sido apontados como fatores determinantes da atual epidemia de obesidade $^{(7-9)}$.

O excesso de tecido adiposo, na região abdominal, é considerado obesidade central, ou andróide, e apresenta risco diferenciado em relação a diversas morbidades, quando comparado a outras formas de distribuição de gordura corporal ${ }^{(10,2)}$. Esta relação pode ser explicada pela alta atividade metabólica do tecido adiposo visceral ${ }^{(11)}$.

A circunferência de cintura (CC) tem sido apontada como a medida antropométrica melhor correlacionada à quantidade de tecido adiposo visceral ${ }^{(10,12)}$.

Segundo Olinto et al. ${ }^{(10)}$, são sugeridos dois níveis de promoção à saúde, de acordo com a medida de CC. O nível I de intervenção inclui medidas de prevenção primária, ou seja, ações visando a perda de peso, a prática de atividade física, a cessação do hábito de fumar e a inclusão de outros hábitos de vida mais saudáveis. O nível II de intervenção inclui medidas de prevenção secundária, ou seja, o acompanhamento dos indivíduos por profissionais da área da saúde, visando redução de massa corporal e conseqüente diminuição de risco.
A atividade física é o componente mais variável do gasto energético diário ${ }^{(4)}$. Tendo em vista o impacto desta variável sobre o balanço energético, a atividade física pode contribuir para a prevenção do ganho de peso e para o tratamento da obesidade, podendo ter um importante papel para a diminuição do crescimento nas taxas de obesidade ${ }^{(13,14,4)}$

Devido à importante contribuição da atividade física no balanço energético, o presente estudo visa correlacionar o nível de CC com o grau de atividade física, buscando verificar se indivíduos mais ativos fisicamente tendem a ter menores valores de CC.

\section{METODOLOGIA}

A amostra foi selecionada de forma aleatória, sendo composta por 40 indivíduos, 16 do gênero feminino e 24 do gênero masculino, com faixa etária de 18 a 44 anos, os quais aceitaram participar da pesquisa e assinaram o termo de consentimento livre e esclarecido. O estudo foi aprovado pelo Comitê de Ética em Pesquisa da Universidade Estácio de Sá - RJ.

A coleta foi realizada durante três dias consecutivos (15, 16 e 17 de março de 2007), sendo feita sempre nos mesmos horários, durante os três dias, no Barra Shopping, localizado na Barra da Tijuca, bairro da zona oeste do Município do Rio de Janeiro.

A CC foi medida com uma trena antropométrica Sanny ${ }^{\circledR}$ (precisão de $0,1 \mathrm{~cm}$ ), diretamente sobre a pele, no ponto médio entre a crista ilíaca e o rebordo costal inferior, conforme proposto pela I Diretriz Brasileira de Diagnóstico e Tratamento da Síndrome Metabólica ${ }^{(12)}$.

A massa corporal foi mensurada em uma balança digital Tanita ${ }^{\circledR}$ (precisão de $0,1 \mathrm{~kg}$ ), estando os indivíduos descalços e com o mínimo de vestimentas possíveis. A altura foi avaliada sob as mesmas condições da avaliação da massa corporal, em uma balança com estadiômetro Filizola® (precisão de $0,5 \mathrm{~cm}$ ), com cursor em ângulo de $90^{\circ}$ em relação à escala, estando o avaliado em posição ereta, com os pés unidos, braços estendidos ao lado do corpo e cabeça posicionada segundo o plano de Frankfurt.

Com os resultados da massa corporal e da altura destes indivíduos, foi calculado o índice de massa corporal (IMC), definido como a massa corporal, em quilogramas, dividida pelo quadrado da altura, em metros $\left(\mathrm{kg} / \mathrm{m}^{2}\right)$. O IMC foi utilizado pelo fato de possuir uma forte correlação com o 
percentual de gordura corporal em grandes populações ${ }^{(5)}$. Além disso, utilizou-se esta fórmula pois há uma relação entre IMC e riscos à saúde $(15,16)$.

Os resultados do IMC foram interpretados de acordo com os critérios de diagnóstico da Organização Pan-americana de Saúde ${ }^{(8)}$, onde: os indivíduos com valores de 18,5 a $24,9 \mathrm{~kg} / \mathrm{m}^{2}$ foram considerados eutróficos; de 25,0 a $29,9 \mathrm{~kg} / \mathrm{m}^{2}$ foram classificados como possuindo sobrepeso; e valores superiores a $30 \mathrm{~kg} / \mathrm{m}^{2}$, obesidade.

O nível de $\mathrm{CC}$ foi classificado de acordo com os níveis de intervenção: nível I para mulheres com CC de 80 a $88 \mathrm{~cm}$ e homens com CC de 94 a $102 \mathrm{~cm}$; e nível II para mulheres com CC > $88 \mathrm{~cm}$ e homens com CC > $102 \mathrm{~cm}$. Foram considerados como adiposidade abdominal adequada, os valores de $\mathrm{CC}<80$ e $94 \mathrm{~cm}$ para mulheres e homens, respectivamente ${ }^{(10)}$.

O grau de atividade física foi avaliado através do questionário de atividades físicas habituais, desenvolvido originalmente por Russell R. Pate, da University of South Carolina (Estados Unidos), traduzido e adaptado por Nahas ${ }^{(17)}$. O grau de atividade física foi classificado de acordo com o somatório de pontos assinalados no questionário de atividades físicas habituais, sendo os indivíduos classificados como: inativos, pouco ativos, moderadamente ativos ou muito ativos.

Para avaliação do estilo de vida, utilizou-se o pentáculo do bem-estar (18), que abrange os componentes relacionados à nutrição, à atividade física, ao comportamento preventivo, aos relacionamentos e ao controle do estresse. Para tanto, considerou-se a seguinte escala: 0 e 1 = perfil negativo, 2 e 3 = perfil positivo. 0 pentáculo do bem-estar foi utilizado no intuito de se avaliar uma possível influência do estilo de vida sobre os níveis de CC e de IMC.

Para análise descritiva dos dados, utilizou-se a média, como medida de tendência central, e o desvio-padrão, para descrever a variabilidade dos resultados obtidos, além das porcentagens para avaliar a prevalência de sobrepeso, obesidade, CC nível I e nível II, em ambos os gêneros. Foi aplicado o teste t de Student para amostras independentes, visando encontrar as diferenças entre os gêneros, sendo considerados significantes os valores de $p \leq 0,05$. Foi utilizada a correlação de Pearson para encontrar a correlação entre o nível de CC e o grau de atividade física, assim como para encontrar a relação entre IMC e os valores de CC. A análise dos dados foi realizada mediante a utilização do programa estatístico Statistical Package for the Social Science (SPSS ${ }^{\circledR}$ ), versão 12.0 .

\section{RESULTADOS}

Dos 40 indivíduos avaliados, $60 \%$ foram do gênero masculino e $40 \%$ do gênero feminino.

Os homens mostraram-se mais altos e pesados do que as mulheres $(p \leq 0,05)$, entretanto, não houve diferença estatisticamente significativa entre os valores de IMC do gênero masculino e feminino $(p=0,456)$.

Os dados relativos à idade e aos indicadores antropométricos, possuindo a média, desvio padrão de ambos os gêneros e o valor de $p$, referente aos resultados do teste t de Student, utilizado para comparação entre os gêneros, estão expostos na TABELA 1.

Utilizando-se os critérios de diagnóstico da OPAS ${ }^{(8)}$, foi encontrada uma prevalência de $45 \%$ de sobrepeso e $12,5 \%$ de obesidade. Deste total em relação ao sobrepeso, apenas $22,2 \%$ foram do gênero feminino, enquanto que o

\section{TABELA 1}

DISTRIBUIÇÃO DA MÉDIA E DESVIO-PADRÃO DA IDADE E DOS INDICADORES ANTROPOMÉTRICOS DE ACORDO COM O GÊNERO ( $\mathrm{N}=40)$.

\begin{tabular}{lccc}
\hline Variáveis & Homens $(\mathbf{n}=\mathbf{2 4})$ & Mulheres $\mathbf{( n = 1 6 )}$ & Valor $\mathbf{p}$ \\
\hline Idade (anos) & $30,63 \pm 6,52$ & $29,63 \pm 10,16$ & 0,73 \\
Massa Corporal $(\mathrm{kg})$ & $81,81 \pm 12,17$ & $64,74 \pm 13,29$ & 0,000 * \\
Estatura $(\mathrm{m})$ & $1,75 \pm 0,06$ & $1,61 \pm 0,07$ & 0,000 * \\
IMC $\left(\mathrm{kg} / \mathrm{m}^{2}\right)$ & $26,73 \pm 3,64$ & $25,34 \pm 6,36$ & 0,456 \\
CC $(\mathrm{cm})$ & $88,48 \pm 11,04$ & $78,79 \pm 14,85$ & 0,035 * \\
\hline
\end{tabular}


TABELA 2

PREVALÊNCIA DE SOBREPESO E OBESIDADE ENTRE OS GÊNEROS ( $\mathrm{N}=40)$.

\begin{tabular}{lcc}
\hline Classificação: & Homens $(\mathbf{n = 2 4})$ & Mulheres(n=16) \\
\hline & $\%$ & $\%$ \\
\cline { 2 - 3 } Normal & 29,17 & 62,5 \\
Sobrepeso & 58,33 & 25 \\
Obesidade & 12,5 & 12,5 \\
\hline
\end{tabular}

gênero masculino foi responsável por $77,8 \%$. Já em relação à obesidade, $60 \%$ foram provenientes de indivíduos do gênero masculino e $40 \%$ por indivíduos do gênero feminino.

Os dados da prevalência de sobrepeso e obesidade, observados através dos valores de IMC entre os gêneros, estão expostos na TABELA 2.

Foi observada uma alta prevalência dos níveis I e II de intervenção, de acordo com a medida de CC, tanto em homens, quanto em mulheres, aonde $37,5 \%$ das mulheres e $33,32 \%$ dos homens foram classificados como possuindo CC nível I ou nível II.

Os dados da prevalência dos níveis de intervenção, baseado nos valores de CC, estão expostos na TABELA 3.

Considerando os resultados encontrados no grau de atividade física, observados através do questionário de atividades físicas habituais, foi encontrado um maior índice de inatividade física entre as mulheres, em relação aos homens, uma vez que $31,25 \%$ das mulheres e $20,83 \%$ dos homens foram classificados como inativos fisicamente.

A TABELA 4 expõe os dados do grau de atividade física entre os gêneros, através do questionário de atividades físicas habituais.
O nível de CC correlacionou-se negativamente com o grau de atividade física, tanto em homens $(r=-0,55$; $p<0,01)$, quanto em mulheres $(r=-0,55 ; p<0,05)$.

Observou-se uma forte correlação positiva entre o IMC e o nível de CC, tanto entre homens $(r=0,82 ; p<0,01)$, quanto entre mulheres $(r=0,95 ; p<0,01)$.

Conforme apresentado no GRÁFICO 1, os homens apresentaram um perfil negativo do componente preventivo, enquanto que as mulheres apresentaram um perfil positivo desse componente, diferindo, significativamente, entre os gêneros $(p \leq 0,05)$. No componente controle do estresse, houve uma diferença estatisticamente significativa entre os gêneros $(p \leq 0,05)$, tendo os homens adquirido um perfil positivo para esse componente e as mulheres, um perfil negativo.

Nos componentes nutrição e atividade física, ambos os gêneros tiveram um perfil negativo, não havendo diferenças significativas entre homens e mulheres. $O$ componente relacionamento apresentou um perfil positivo, nos dois gêneros, sem diferença significativa entre estes.

\section{DISCUSSÃO}

Apesar deste estudo não ter representatividade populacional e ter verificado apenas características específicas dos freqüentadores do shopping, os dados em

\section{TABELA 3}

\section{PREVALÊNCIA DOS NÍVEIS DE CIRCUNFERÊNCIA DE CINTURA ENTRE} OS GÊNEROS $(\mathrm{N}=40)$.

\begin{tabular}{lcc}
\hline Classificação & Homens $(\mathbf{n}=\mathbf{2 4})$ & Mulheres $(\mathbf{n}=\mathbf{1 6})$ \\
\hline & $\%$ & $\%$ \\
\cline { 2 - 3 } Normal & 66,67 & 62,5 \\
Nível I & 16,67 & 18,75 \\
Nível II & 16,67 & 18,75 \\
\hline
\end{tabular}


TABELA 4

ANÁLISE DO GRAU DE ATIVIDADE FÍSICA ENTRE OS GÊNEROS, OBTIDOS ATRAVÉS DOS RESULTADOS DO QUESTIONÁRIO DE ATIVIDADES FÍSICAS HABITUAIS ( $\mathrm{N}=40)$.

\begin{tabular}{lcc}
\hline Classificação: & Homens $(\mathbf{n}=\mathbf{2 4})$ & Mulheres $(\mathbf{n}=\mathbf{1 6})$ \\
\hline & $\%$ & $\%$ \\
\cline { 2 - 3 } Inativo & 20,83 & 31,25 \\
Pouco Ativo & 16,67 & 6,25 \\
Moderadamente Ativo & 20,83 & 18,75 \\
Muito Ativo & 41,67 & 43,75 \\
\hline
\end{tabular}

\section{GRÁFICO 1 \\ COMPARAÇÃO DO PERFIL DO ESTILO DE VIDA ENTRE OS GEENEROS, AVALIADO ATRAVÉS DO QUESTIONÁRIO O PENTÁCULO DO BEM-ESTAR.}

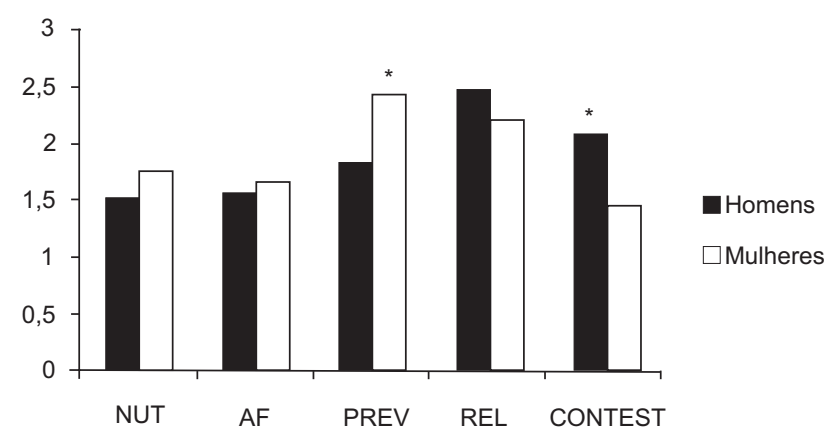

Os dados são referentes às médias dos componentes: nutrição (NUT), atividade física (AF), preventivo (PREV), relacionamento (REL) e controle do estresse (CONT EST) do questionário "O Pentáculo do Bem-estar", em homens e mulheres.

$$
{ }^{*} p \leq 0,05
$$

relação à obesidade são semelhantes aos encontrados no Estado do Rio de Janeiro ${ }^{(19,20)}$.

Entretanto, deve-se atentar para a elevada prevalência de sobrepeso observada na presente amostra (45\%), enquanto que a média encontrada no Estado do Rio de Janeiro foi de $33,5 \%{ }^{(19)}$. Assim, nos resultados deste trabalho, a prevalência de sobrepeso foi superior no gênero masculino, em relação ao gênero feminino, não sendo encontradas diferenças significativas entre a prevalência de obesidade entre os gêneros ${ }^{(19)}$.

Em pesquisa recente, Mariath et al. ${ }^{(21)}$ constataram que os homens possuíam uma probabilidade quase três vezes maior de apresentarem sobrepeso ou obesidade, se relacionados a indivíduos do gênero feminino, explicando, assim, possivelmente, a maior prevalência de sobrepeso entre o gênero masculino observada nos resultados deste estudo.

Mariath et al. ${ }^{(21)}$, ainda, encontraram uma probabilidade duas vezes maior de indivíduos com sobrepeso apresentarem aumentos na pressão arterial sistólica (PAS) e na pressão arterial diastólica (PAD), em relação aos indivíduos eutróficos. Já indivíduos com obesidade, apresentaram uma probabilidade quatro vezes maior de apresentarem aumentos na PAS e cinco vezes maior de apresentarem aumentos em PAD, em relação aos indivíduos eutróficos.

Estes resultados se tornam preocupantes, uma vez que, na presente amostra, foi apresentado um alto índice de sobrepeso e obesidade entre o gênero masculino, pois apenas $29,17 \%$ dos indivíduos avaliados foram classificados como eutróficos.

Quando analisados os níveis de CC, as mulheres obtiveram maior prevalência de CC nível I e CC nível II, se comparadas ao gênero masculino, estando de acordo com os dados encontrados na literatura ${ }^{(10,22)}$.

A elevada prevalência encontrada, em ambos os gêneros, para CC nível I e CC nível II apresenta-se como um dado preocupante, pois a CC foi apresentada como um bom preditor para o perfil lipídico em homens, apresentando uma associação independente para hipertensão e diabetes em mulheres ${ }^{(23,24)}$.

Souza-e-Sá Júnior et al. ${ }^{(25)}$, estudando militares do gênero masculino, com sobrepeso, encontraram uma correlação direta entre a CC e o colesterol total e LDL-C . 
Em pesquisa avaliando a prática de atividade física de lazer, em 3740 funcionários técnico-administrativos, de ambos os gêneros, em uma universidade localizada no Estado do Rio de Janeiro, foi encontrada uma alta taxa de inatividade física no tempo destinado ao lazer, uma vez que apenas $40,8 \%$ das mulheres e $52 \%$ dos homens referiram realizar alguma prática de atividade física nestes momentos (26). Gomes, Siqueira e Sichieri ${ }^{(14)}$, ao avaliarem a prática de atividade física de lazer, em 4331 indivíduos, de ambos os gêneros, do Município do Rio de Janeiro, encontraram que $59,8 \%$ dos homens e $77,8 \%$ das mulheres relataram nunca realizar atividade física de lazer.

Os resultados destas duas pesquisas vão de encontro ao do presente estudo, em relação a um maior índice de sedentarismo entre as mulheres, do que entre os homens. Contudo, a prevalência de inatividade física, encontrada nos dois estudos relatados, foi superior ao encontrado por esta pesquisa, podendo a grande diferença entre estes resultados ter sido influenciada pela forma de classificação do grau de atividade física.

Segundo Trombeta ${ }^{(9)}$, os indivíduos que se mantêm fisicamente ativos, ao longo da vida, têm menos chances de se tornarem obesos, além de possuírem melhor distribuição de gordura corporal, com menos depósitos de gordura intra-abdominal.

De fato, corroborando os achados do presente trabalho, Olinto et al. ${ }^{(10)}$ observaram uma associação inversa entre a obesidade abdominal e a atividade física. Czernichow et al. ${ }^{(27)}$, estudando a relação entre a adiposidade central e a prática de atividade física, afimaram que a probabilidade dos indivíduos da amostra virem a obter maiores valores de razão cintura-quadril era aumentada com a inatividade física.

Silva et al. ${ }^{(28)}$ observaram uma correlação negativa entre $\mathrm{CC}$ e $\mathrm{VO}_{2 m a ́ x}$, tanto em homens $(r=-0,49)$, quanto em mulheres $(r=-0,58)$. Além disso, no referido estudo, o $\mathrm{VO}_{2 \text { máx }}$ correlacionou-se negativamente com os níveis de pressão arterial, em ambos os gêneros, bem como com a taxa de triglicerídeos, em mulheres, e com resistência à insulina, em homens, além de apresentar uma associação positiva com o HDL-c, em homens.

A forte correlação positiva encontrada neste estudo, entre IMC e os valores de CC, torna-se interessante, uma vez que Souza et al. ${ }^{(29)}$ observaram que os indivíduos obesos, principalmente aqueles com excesso de adiposidade abdominal, apresentavam maior prevalência de hipertensão arterial, diabetes mellitus e dislipidemia, se relacionados à população normal.

Em estudo realizado com nipo-brasileiros, por Lerario et al. ${ }^{(30)}$, foi observada uma maior prevalência de hipertensão arterial, de dislipidemia e de diabetes mellitus, em indivíduos com excesso de massa corporal e com excesso de adiposidade abdominal.

Rezende et al. ${ }^{(22)}$ verificaram que, com o aumento do IMC e da gordura na região abdominal, houve, principalmente, elevação da glicemia de jejum, dos níveis de triglicérides, redução dos níveis de HDL-c e elevação da pressão arterial.

Segundo Olinto et al. ${ }^{(24)}$ os riscos para desenvolvimento de hipertensão e diabetes possuem uma associação direta com IMC e CC.

Silva et al. ${ }^{(28)}$ relatam uma associação significativa entre os valores, tanto do IMC, quanto da CC, com os níveis de pressão arterial, de lipídios-lipoproteína plasmáticas e com a sensibilidade insulínica.

O perfil nutricional negativo, encontrado nestes freqüentadores do Barra Shopping, possivelmente, foi influenciado pela grande oferta de lanchonetes e restaurantes, no local, servindo alimentos altamente calóricos e ricos em gordura. A associação do perfil nutricional negativo com o perfil de atividade física negativo parece ter sido responsável pelos elevados índices encontrados de IMC e CC da amostra estudada.

Dentro das limitações do estudo, a principal é referente ao tamanho da amostra, que pode ser justificada pelo fato desta ter sido selecionada de forma aleatória, dependendo da disponibilidade e da aceitação dos freqüentadores para se tornarem voluntários.

\section{CONCLUSÃO}

O estilo de vida dos indivíduos estudados parece ter exercido uma forte influência sobre os resultados de sobrepeso, de obesidade, de CC nível I e nível II, uma vez que foi observado um perfil negativo, em ambos os gêneros, nos componentes nutrição e atividade física. Apesar de ter sido encontrada apenas uma correlação média negativa entre o nível de CC e o grau de atividade física, esta foi significativa, tanto em homens, quanto em mulheres, sugerindo que o grau de atividade física possa influenciar, ao menos em parte, os valores de CC. 
Revista de Educação Física 2008 Set; 142:42-49. Rio de Janeiro (RJ) - Brasil.

Novos estudos devem ser realizados, buscando um número maior de voluntários e, se possível, comparando shoppings localizados em diferentes regiões, atingindo as diversas camadas sociais da população.

\section{REFERÊNCIAS BIBLIOGRÁFICAS}

1. Ferreira S, Tinoco ALA, Panato E, Viana NL. Aspectos etiológicos e o papel do exercício físico na prevenção e controle da obesidade. Revista de Educação Física 2006; 133: 15-24.

2. Pereira LO, Francischi RP, Lancha JR AH. Obesidade: hábitos nutricionais, sedentarismo e resistência à insulina. Arq Bras Endocrinol Metabol 2003; 47(2): 111-27.

3. Suárez MMS, Astoviza MB, Puig MEL. Obesidad: tratamiento no farmacológico y prevención. Rev Cubana Endocrinol 2002; 13(1): 35-42.

4. Jakicic JM, Otto AD. Physical activity considerations for the treatment and prevention of obesity. Am J Clin Nutr 2005; 82 (Suppl): 226S-9S.

5. Nammi S, Koka S, Chinnala K, Boini KM. Obesity: an overview on its current perspectives and treatment options. Nutrition Journal 2004; 3(3).

6. Martins FR, Rodrigues dos Santos JA. Atividade física de lazer, alimentação e composição corporal. Rev Bras Educ Fís Esp 2004; 18 (2): 159-67.

7. Mendonça CP, Anjos LA. Aspectos das práticas alimentares e da atividade física como determinantes do crescimento do sobrepeso/obesidade no Brasil. Cad Saúde Pública 2004; 20(3): 698-709.

8. Organização Pan-Americana da Saúde (OPAS). Doenças crônico-degenerativas e obesidade: estratégia mundial sobre alimentação saudável, atividade física e saúde, 2003 [on-line]. Disponível em: URL: http://www.opas.org.br/ sistema/arquivos/d_cronic.pdf(21 out.2006).

9. Trombeta IC. Exercício físico e dieta hipocalórica para o paciente obeso: vantagens e desvantagens. Rev Bras Hipertens 2003; 10(2): 130-3.

10. Olinto MTA, Nacul LC, Costa JSD, Gigante DP, Menezes AMB, Macedo S. Níveis de intervenção para obesidade abdominal: prevalência e fatores associados. Cad Saúde Pública 2006; 22(6): 1207-15.

11. Hermsdorff HHM, Monteiro JBR. Gordura visceral, subcutânea ou intramuscular: onde está o problema? Arq Bras de Endocrinol Metabol 2004; 48 (6): 803-11.

12. Sociedade Brasileira de Hipertensão, Sociedade Brasileira de Cardiologia, Sociedade Brasileira de Endocrinologia e Metabologia, Sociedade Brasileira de Diabetes, Associação Brasileira para Estudos da Obesidade. I Diretriz brasileira de diagnóstico e tratamento da Síndrome Metabólica. Arq Bras Cardiol 2005; 84(Suppl.1): 3-28.

13. Ciolac EG, Guimarães GV. Exercício físico e síndrome metabólica. Rev Bras Med Esporte 2004; 10(4): 319-24.

14. Gomes VB, Siqueira KS, Sichieri R. Atividade física em uma amostra probabilística da população do Município do Rio de Janeiro. Cad Saúde Pública 2001; 17 (4): 969-76.

15. American College of Sports Medicine (ACSM). Position stand: appropriate intervention strategies for weight loss and prevention of weight regain. Med Sci Sports Exerc 2001; 33(12): 2145-56.

16. Brown CD, Higgins M, Donato KA, Rohde FC, Garrison R, Obarzanek E, et al. Body mass index and the prevalence of hypertension and dyslipidemia. Obesity Res 2000; 8(9): 605-19.

17. Nahas MV. Atividade física, saúde e qualidade de vida: conceitos e sugestões para um estilo de vida ativo. $4^{\underline{a}}$ ed. Londrina: Midiograf; 2006. 
18. Nahas MV, Barros MVG, Francalacci VL. O pentáculo do bem-estar: base conceitual para avaliação do estilo de vida de indivíduos ou grupos. Revista Brasileira de Atividade Física e Saúde 2000; 5(2): 48-59.

19. INCA. Inquérito domiciliar sobre comportamentos de risco e morbidade referida de doenças e agravos não transmissíveis: Brasil, 15 capitais e Distrito Federal, 2002 - 2003, 2004 [on-line]. Disponível em: URL: http://www.inca.gov.br/inquerito/docs/completa.pdf(02 mai.2007).

20. Sichieri S. Dietary patterns and their associations with obesity in the Brazilian city of Rio de Janeiro. Obesity Res 2002; 10(1): 42-8.

21. Mariath AB, Grillo LP, Da Silva RO, Schmitz P, De Campos IC, Medina JRP, et al. Obesidade e fatores de risco para o desenvolvimento de doenças crônicas não-transmissíveis entre usuários de unidade de alimentação e nutrição. Cad Saúde Pública 2007; 24 (4): 897-905.

22. Rezende FAC, Rosado LEFPL, Ribeiro RCL, Vidigal FC, Vasques ACJ, Bonard IS, et al. Índice de massa corporal e circunferência abdominal: associação com fatores de risco cardiovascular. Arq Bras Cardiol 2006; 87(6): 728-34.

23. Lemos-Santos MGF, Valente JG, Gonçalves-Silva RMV, Sichieri R. Waist circumference and waist-to-hip ratio as predictors of serum concentration of lipids in Brazilian men. Nutrition 2004; 20: 857-62.

24. Olinto MTA, Nacul LC, Costa JSD, Gigante DP, Menezes AMB, Macedo S. Waist circumference as a determinant of hypertension and diabetes in Brazilian women: a population-based study. Public Health Nutrition 2004; 7(5): 629-35.

25. Souza-e-Sá Júnior JM, Monteiro-Gomes MA, Alcaraz CCP, De Sousa JCM, De Souza FH, Da Silva CTF, et al. Relação de medidas antropométricas e fatores de risco cardiovasculares. Revista de Educação Física 2007; 136: 38-46.

26. Salles-Costa R, Heilborn ML, Werneck GL, Faerstein E, Lopes CS. Gênero e prática de atividade física de lazer. Cad Saúde Pública 2003; 19(Suppl. 2): S325-33.

27. Czernichow S, Bertrais S, Preziosi P, Galan P, Hercberg S, Oppert JM. Indicators of abdominal adiposity in middle-aged participants of the SU.VI.MAX study: relationships with educational level, smoking status and physical inactivity. Diabetes Metab 2004; 30(2): 153-9.

28. Silva JLT, Barbosa DS, Oliveira JA, Guedes DP. Distribuição centrípeta da gordura corporal, sobrepeso e aptidão cardiorrespiratória: associação com sensibilidade insulínica e alterações metabólicas. Arq Bras Endocrinol Metabol 2006; 50(6): 1034-40.

29. Souza LJ, Neto CG, Chalita FEB, Reis AFF, Bastos DA, Souto JTD, et al. Prevalência de obesidade e fatores de risco cardiovascular em Campos, Rio de Janeiro. Arq Bras Endocrinol Metab 2003; 47(6): 669-76.

30. Lerario DDG, Gimeno SG, Franco LJ, Lunes M, Ferreira SRG. Excesso de peso e gordura abdominal para a síndrome metabólica em nipo-brasileiros. Rev Saúde Pública 2002; 36 (1): 4-11.

\section{Endereço para correspondência:}

Marcus Vinicius Accetta Vianna

R. Gen. Pereira da Silva, no 87 apt 1406

Icaraí - Niterói - RJ

CEP: 24220-030

e-mail: marcusvianna@yahoo.com.br 